\title{
INTERPRETIVE SUMMARIES, MARCH 2013
}

Effects of supercritical fluid extraction pressure on chemical composition, microbial population, polar lipid profile, and microstructure of goat cheese. By Sánchez-Macías et al., page 1325. The use of low-fat goat milk in cheese-making results in a product with inadequate texture and unappealing sensory attributes. Proteolysis and lipolysis are also altered during the ripening; therefore, new technologies are required to avoid these disadvantages. Supercritical fluid extraction is a novel "green" method used to remove lipids after cheese is ripened, while keeping all the attributes of full-fat cheese. We report the effects of varying pressures during supercritical fluid extraction with $\mathrm{CO}_{2}$ on chemical composition, lipid profile, microstructure, and the microbial population of 2 varieties of ripened goat cheeses. This method is a good alternative to produce goat cheese with less fat, specifically triglycerides and cholesterol, and lower microbial load after maturation.

Quantitative measurement of vitamin $K_{2}$ (menaquinones) in various fermented dairy products using a reliable high-performance liquid chromatography method. By Manoury et al., page 1335 . Vitamin $\mathrm{K}_{2}$, mainly present in fermented food, is increasingly studied for its health benefits, particularly for cardiovascular and bone health. Quantitative dosages of vitamin $\mathrm{K}_{2}$ in different fermented dairy products (thermophilic cheeses, mesophilic cheeses, and fresh fermented milks) are presented. Abundance of vitamin $\mathrm{K}_{2}$ forms according to different fermented dairy products types is discussed.

Skimmed, sterilized, and concentrated bovine late colostrum promotes both prevention and recovery from intestinal tissue damage in mice. By Cairangzhuoma et al., page 134\%. Colostrum, a nutrient-rich fluid produced by female mammals immediately after giving birth, is loaded with several immune, growth, and tissue repair factors. Our study revealed that colostrum was effective for healing and inhibition of small intestinal inflammations induced by drugs. The effective ingredient was casein, which is also main ingredient of cheese.

Optical backscatter method for determining thermal denaturation of $\beta$-lactoglobulin and other whey proteins in milk. By Lamb et al., page 1356. United States dairy products have an influential role in the American diet and increasing demand abroad as exports. With such demand, efficiencies and inefficiencies in dairy processing can have a large effect on company profit and market prices. The heat denaturation of whey proteins affects the functional properties of milk for processing. The purpose of this study was to establish if an optical backscatter response of whey denaturation, particularly $\beta$-lactoglobulin, during milk heat treatment could be determined. This would be the basis for an inline optical measurement technology.

Influence of heating and acidification on the flavor of whey protein isolate. By White et al., page 1366. Whey protein manufacture influences the flavor of dried whey protein. When whey protein isolate (WPI; $\geq 90 \%$ protein) is used in beverages, it is often instantized, acidified, and heated. The purpose of this study was to determine the effect of instantization, acidification, and heating on WPI flavor. Flavors and volatile compounds specific to instantized WPI were documented but these effects were small compared with the effects of acidification and heating. Acidification and heating negatively affected the flavor of regular and instantized WPI, promoting off-flavors and increased concentrations of aldehydes, ketones, and sulfur compounds.

Use of potassium chloride and flavor enhancers in low sodium Cheddar cheese. By Grummer et al., page 1401. Low sodium Cheddar cheese was produced using 2 potassium chloride sources as salt replacers, and flavor enhancers were used to modify flavor. Cheese chemistry and sensory attributes were analyzed over 4 mo of aging, and a consumer acceptability panel was conducted at 6 mo of age. Consumer acceptability of low sodium cheeses was not different from that of the full sodium control. Differences in cheese chemistry and sensory attributes existed as a result of the salt replacers and cheese-make procedure. At the levels used, flavor enhancers had relatively minor effects.

Ameliorative potential of whey protein hydrolysate against paracetamol-induced oxidative stress. By Athira et al., page 1431. The objective of the study was to evaluate the potential protective effect of whey protein hydrolysate (WPH) against paracetamolinduced oxidative stress, which causes hepatic and renal toxicity. The WPH successfully mitigated the increase in the concentration of oxidative biomarkers in serum and restored activities of antioxidant enzymes in liver as well as levels of blood urea nitrogen in mice with paracetamol-induced oxidative stress. The WPH also reduced the extent of lipid peroxidation in the liver of these mice. Therefore, we conclude that WPH could be used as an effective protector against paracetamolinduced hepato-nephrotoxicity.

Antimicrobial potential for the combination of bovine lactoferrin or its hydrolysate with lactoferrin-resistant probiotics against foodborne pathogens. By Chen et al., page 1438. This study described 8 probiotic strains that can resist the 
antibacterial activity of the apo form of bovine lactoferrin (apo-bLF) and its pepsin-digested hydrolysate (bLF-hydrolysate). A combination of apo-bLf or bLfhydrolysate with specific probiotics led to synergistic or partially synergistic effects against the growth of foodborne pathogens. This study highlights the possibility of using apo-bLf or its hydrolysate with specific probiotic activity against pathogen infection and for use in prophylactic supplements.

Development of a method to determine essential oil residues in cow milk. By Hallier et al., page $144 \%$. The aim of our study was to develop a reliable technique capable of quantifying the presence of residues of essential oils in unprocessed cow milk. This technique is based on solid-phase microextraction coupled with gas chromatography-mass spectrometry. Several parameters were optimized through their effect on the sensitivity of the method. The methodology developed was then successfully applied to milk samples capable of containing components of the essential oils most used in breeding. The project's importance is to guarantee that essential oils with a positive effect on cow breeding have no negative effects on milk or derived products.

Lactobacillus helveticus as a tool to change proteolysis and functionality in Swiss-type cheeses. By Sadat-Mekmene et al., page 1455. Lactic acid bacteria play a crucial role in the texture, aroma, and health properties of various dairy products. Lactobacillus helveticus species possess at least 2 cell wall proteinases to efficiently hydrolyze milk proteins. We aimed to deepen knowledge on these proteinases regarding their expression in cheese. This study investigated whether the type of proteinases and their number can change the final functional properties of Swiss-type cheese in terms of stretching.

Short communication: Radio frequency dielectric heating of nonfat dry milk affects solubility and whey protein nitrogen index. By Chen et al., page 1471. Dried dairy powders may pose a significant food safety risk. Radio frequency dielectric heating (RFDH) has potential as a postprocess lethality treatment for nonfat dry milk (NDM), but the heat may alter the functional properties. High-heat $(\mathrm{HH})$ and low-heat (LH) NDM were processed by RFDH and products were assessed for whey protein nitrogen index (WPNI) and solubility. Processing at $\geq 80^{\circ} \mathrm{C}$ decreased WPNI and solubility in LH-NDM, but only decreased solubility in HH-NDM. Thus, RFDH should be investigated further to determine its application as a pathogen-control process for NDM while maintaining acceptable functional properties.

Investigating a dilution effect between somatic cell count and milk yield and estimating milk production losses in Irish dairy cattle. By Boland et al., page 1477. Increased somatic cell counts (SCC) are associated with reduced milk yield. Additionally, it has been hypothesized that, as milk yield increases, SCC are diluted in cattle without an intramammary infection. In this study, various adjustments were used to investigate this hypothesis. Further, lactation milk production losses with increasing SCC were estimated. We found no evidence of a dilution effect in the association between SCC and milk yield in Irish dairy cattle. Milk production losses increased with increasing SCC and parity.

Factors associated with fertility outcomes in cows treated with protocols to synchronize estrus and ovulation in seasonal-calving, pasturebased dairy production systems. By Herlihy et al., page 1485. Factors associated with fertility outcomes in lactating dairy cows treated with protocols to synchronize estrus and ovulation in seasonal-calving, pasturebased dairy production systems were investigated. These factors included ovarian follicle and corpus luteum status at protocol initiation, parity, milk production, body condition score, progesterone on d 11 after insemination, and days in milk. Presence of a corpus luteum at the onset of synchronization, greater body condition score at the time of artificial insemination, and greater days in milk were associated with increased likelihood of synchronization, submission for insemination, conception at first service, and pregnancy at first service in cows treated with protocols to synchronize estrus and ovulation

Unraveling the microbiota of teat apices of clinically healthy lactating dairy cows, with special emphasis on coagulase-negative staphylococci. By Braem et al., page 1499. The teat apices of lactating cows on 2 well-managed Flemish dairy herds exhibited a wide bacterial diversity between milking rounds. A combination of culture-dependent and culture-independent methodologies applied on the microbiota of the teat apices indicated the presence of 20 bacterial genera from 3 phyla, with the most prevalent bacterial group being the coagulase-negative staphylococci. Although no evidence was found for an association between teat microbiota and udder health in this study, more insight into the teat microbiota may contribute to development of additional udder health management methods.

The effect of administering ketoprofen on the physiology and behavior of dairy cows following surgery to correct a left displaced abomasum. By Newby et al., page 1511. Few dairy cattle are provided pain management following left displaced abomasum (LDA) surgery. The purpose of this field trial was to examine the effects of providing the pain medication ketoprofen to dairy cattle immediately following LDA 
surgery performed on the right flank or by ventral approach. Ketoprofen had little effect on the physiological measures. However, cows that had surgery by the ventral approach spent significantly less time lying down compared with those that had surgery on the right flank, potentially indicating greater postsurgical pain. Producers also reported that cows that received ketoprofen had improved appetite compared with cows provided saline.

Investigating associations between milk metabolite profiles and milk traits of Holstein cows. By Melzer et al., page 1521. Milk metabolite profiles are investigated as promising molecular traits specifying the metabolic status of a cow. Studying 1,305 metabolite profiles of Holstein cows and their associations to influencing factors and traditional milk traits will enable insights into their biological role and environmental interrelations. Machine-learning methods, together with feature selection, were applied to reveal groups of metabolites associated with milk traits. Uracil, followed by lactic acid, showed the highest association to somatic cell score (SCS). Also, specific metabolite groups showed negative correlations to lactose and positive correlations to SCS. Some of these metabolites were investigated regarding possible functional interrelations.

The role of extensionists in Santa Catarina, Brazil, in the adoption and rejection of providing pain relief to calves for dehorning. $B y$ Hötzel and Sneddon, page 1535. Brazilian extensionists expressed negative attitudes towards recommending practices that minimize pain during and after dehorning dairy calves. These attitudes were underpinned by the belief that calves experience little pain during dehorning and the perception that farmers had negative attitudes towards practices available to minimize pain. Extensionists' attitudes appear to be embedded in the production-focused political and economic agriculture environment and the lack of teaching, research, and legislation regarding farm animal welfare. Advancement of the animal welfare debate in Brazil, combined with education and research in animal welfare science, are needed to empower extensionists to improve farm animal welfare.

Using temperature-sensing reticular boluses to aid in the detection of production diseases in dairy cows. By Adams et al., page 1549. Early identification of sick cows is paramount in the health management of a dairy herd. Reticular temperature monitoring shows promise as a method for identifying changes in core body temperature, and in the early detection of disease on dairy farms. The associations between increases in reticular temperature in cows and the diagnosis of metritis, mastitis, lameness, and pneumonia by dairy personnel were examined. Of the diseases studied, mastitis and pneumonia had strong associations with an increase in reticular temperature within $4 \mathrm{~d}$ of diagnosis. Combined with careful observation, reticular temperature monitoring could improve early disease identification on dairy operations.

Effect of gonadotropin-releasing hormone or prostaglandin $F_{2 \alpha}$-based estrus synchronization programs for first or subsequent artificial insemination in lactating dairy cows. By Bruno et al., page 1556. Synchronization and resynchronization programs have been widely used in dairy cows to improve fertility. Prostaglandin and gonadotropinreleasing hormone-based programs were developed and altered the estrus expression pattern for both first and subsequent artifical insemination (AI) but did not improve pregnancy per AI. However, prostaglandin-based programs shortened the interval between voluntary waiting period and first AI, as well as the interval between AI.

Monitoring herd incidence of intramammary infection in lactating cows using repeated longitudinal somatic cell count measurements. By Dufour and Dohoo, page 1568. The appropriateness of using repeated milk somatic cell count measurements collected during lactation as a substitute to repeated quarter milk bacteriological culture to estimate and monitor the herd lactational intramammary infection incidence rate was investigated. Somatic cell count measurements, especially quarter-milk measurements, were found to be very valuable for monitoring the lactating period incidence rate and its fluctuations over time. Composite milk somatic cell count measurements, however, showed some important limitations for monitoring the herd intramammary infection incidence rate for most groups of organisms.

The effect of alternative testing strategies and bio-exclusion practices on Johne's disease risk in test-negative herds. By More et al., page 1581. Freedom from Johne's disease among test-negative herds is very difficult to prove, for reasons relating to the disease and available diagnostic tests. This work provides quantitative estimates of Johne's disease risk in testnegative herds, using a modelling approach. It is very difficult to achieve a high probability that infection in the herd is either absent or at very low prevalence (less than the design prevalence), reflecting the low sensitivity of available diagnostic tests. Effective bio-exclusion practices, relating to animal introductions, are critical. The results illustrate an output-based approach to herd classification, with potential for national and field applications.

Bovine leukemia virus and cow longevity in Michigan dairy herds. By Bartlett et al., page 1591. 
In a study of 112 Michigan dairy herds, cattle with milk antibodies to bovine leukemia virus (BLV) were at $23 \%$ higher risk of leaving their herds in the approximately $1.6 \mathrm{yr}$ since BLV testing. The survival analysis was adjusted for herd and lactation number, and a dose response was demonstrated: cattle with higher BLV antibody levels were at greater risk of leaving the herd than were cattle with lower values.

The effect of target postgrazing height on sward clover content, herbage yield, and dairy production from grass-white clover pasture. By Phelan et al., page 1598. The effect of postgrazing height (PGH; 4,5 , or $6 \mathrm{~cm}$ ) on sward white clover content, herbage production, and milk production by dairy cows, was investigated over entire grazing seasons (February to November) for $3 \mathrm{yr}$. The PGH treatment did not significantly affect milk production or sward clover content. However, herbage production was higher in the 4-cm treatment compared with the 6 - $\mathrm{cm}$ treatment. A target $\mathrm{PGH}$ of $4 \mathrm{~cm}$ is therefore recommended for grass-clover swards.

Evaluation of reproductive and economic outcomes of dairy heifers inseminated at induced estrus or at fixed time after a 5-day or 7-day progesterone insert-based ovulation synchronization protocol. By Lopes et al., page 1612. Heifers had their estrus synchronized with prostaglandin $\mathrm{F}_{2 \alpha}$ and were inseminated on estrus (AIE) or had their ovulation synchronized with a controlled internal drug release (CIDR) insert for 5 (CIDR5) or 7 (CIDR7) d, prostaglandin $\mathrm{F}_{2 \alpha}$ at CIDR removal, and timed artificial insemination (AI) 53 to $60 \mathrm{~h}$ later. The AIE heifers had greater pregnancy per AI than CIDR5 and CIDR7 heifers. The CIDR5 heifers tended to have greater pregnancy per AI than CIDR7 heifers, likely because the latter were more likely to have spontaneous luteolysis between CIDR insertion and removal. The AIE treatment resulted in improved input cost (cost of synchronization protocol + semen + rearing + replacement) compared with the CIDR5 and CIDR7 treatments because of greater pregnancy rate, reduced cost of synchronization protocol, reduced expenditure with semen, reduced rearing cost, and increased percentage of heifers pregnant at the end of the study.

Effect of moving dairy cows at different stages of labor on behavior during parturition. By Proudfoot et al., page 1638. Cows are sometimes moved from a group pen to an individual pen to calve. The aim here was to determine if moving cows at different stages of labor would affect the progress of labor and behavior. Cows moved with signs of viscous, bloody mucous, or abdominal contractions experienced longer second stages of labor, spent less time lying, and tended to receive more attention from other cows compared with cows moved earlier. A longer second stage of labor was associated with higher inflammation in early lactation. Thus, moving cows too close to calving may interrupt the natural progression of calving.

Use of a split or single prostaglandin $F_{2 \alpha}$ treatment in a 6-day synchronization protocol for nonlactating dairy cows. By Valldecabres-Torres et al., page 164\%. A limitation of fixed-timed breeding is the need for synchronization protocols that require the administration of several treatments (prostaglandin $\mathrm{F}_{2 \alpha}$ and gonadotropin-releasing hormone) to the same cow before artificial insemination. This means an increase in labor costs resulting from the handling of cows for each treatment. This study shows evidence that increasing the dose of prostaglandin $\mathrm{F}_{2 \alpha}$ allows a reduction in the need for one treatment without decreasing the efficacy of a 6 -d artificial insemination synchronization protocol.

Differential cell count as an alternative method to diagnose dairy cow mastitis. By Pilla et al., page 1653. Differential cell count (DCC) can be used to determine the changes in relative cell proportions occurring in diseased mammary glands. Aims of the present study were to test the consistency of DCC results on subsequent days and to establish an effective cutoff value for the diagnosis of mastitis. A cutoff value of 0.495 for logarithmic polymorphonucelar neutrophilic leukctyes:lymphocytes was established. When such value was applied along with SCC to field data, sensitivity and specificity were 97.3 and $92.3 \%$, respectively. This diagnostic method allowed us to identify inflammatory processes in the mammary gland without the need for bacteriological analysis.

Short communication: Effects of serum obtained from dairy cows with low or high body condition score on in vitro embryo development. $\mathrm{By} O b a$ et al., page 1668. Negative energy balance is a possible factor reducing fertility in dairy cows. The addition of serum, low in nonesterified fatty acids (NEFA) concentrations, derived from cows with high body condition score during in vitro culture of presumptive zygotes increased cleavage rates both at 24 and $48 \mathrm{~h}$ after in vitro fertilization compared with control bovine serum, which had high concentrations of NEFA, without affecting overall blastocyst rate. These observations indicate that high NEFA concentrations may adversely affect very early stage embryo development. Furthermore, serum derived from animals under different conditions or nutritional management can be used in the in vitro embryo culture medium to study the effects of physiology and nutrition on embryo development.

Short communication: Relationship between herd intramammary infection incidence, and 
elimination rate during the dry period. $B y D u$ four and Dohoo, page 1672. The relationship between the rate of acquisition of intramammary infections during the dry period and the rate of elimination of these infections was explored in a cohort of 91 Canadian dairy herds. For coagulase-negative staphylococci and Staphylococcus aureus, herds with a relatively low acquisition rate of infections also demonstrated a relatively high rate of elimination of these infections. Such relationship was not observed for other groups of organisms. Results from the current study indicate that dry period elimination and acquisition rates of some organisms might be driven by common mechanisms.

Short communication: The effect of temperature on performance of milk ketone test strips. By Shire et al., page 167\%. Ketosis is a significant concern in the dairy industry and is regarded as one of the most important metabolic disorders. Early detection of the disorder is critical because development to clinical stages may result in decreased feed intake and milk production. Determining the incidence of subclinical ketosis in fresh cows can help monitor the effectiveness of the transition cow program. The ability to detect subclinical ketosis via measurement of $\beta$-hydroxybutyrate in milk under various temperature conditions using the Keto-Test strip is one method for early detection.

Short communication: Differential immunoglobulin transfer during mastitis challenge by pathogen-specific components. By Wellnitz et al., page 1681. This study investigated the effects of the major pathogenic components lipopolysaccharide of Escherichia coli and lipoteichoic acid of Staphylococcus aureus on blood-milk barrier permeability. The transfer of immunoglobulins and lactate from blood into milk was measured during a quantitatively similar level of mammary inflammation, based upon somatic cell count increase. The results show a more efficient transfer of the blood components immunoglobulin $\mathrm{G}_{2}$ and lactate into milk after intramammary challenge with lipopolysaccharide from $E$. coli than with lipoteichoic acid from Staph. aureus. Such a pathogen-specific difference may play some role in the course and severity of mastitis.

The effect of exogenous phytase on degradation of inositol phosphate in dairy cows. By BraskPedersen et al., page 1691. The present study aimed to investigate the effect of supplementing exogenous phytase (none, low, medium, or high) on inositol phosphate degradation in dairy cows. Four lactating Danish Holstein cows fitted with ruminal, duodenal, and ileal cannulas were used. Rumen and total-tract degradation of inositol phosphate was increased when exogenous phytase was added to the mixed feed ration, and this leaves a potential for reducing phosphorus excretion through reduced dietary $\mathrm{P}$. The trial also revealed that exogenous phytase already started degrading the inositol phosphate in the total mixed ration between mixing and time of ingestion.

Feeding canola meal to dairy cows: A metaanalysis on lactational responses. By Martineau et al., page 1701. A meta-analysis was conducted to compare isonitrogenous rations fed to dairy cows where canola meal was substituted by another protein source. Yields of milk and milk protein and protein percentage, efficiency in milk production (energy-corrected milk/ dry matter intake), and apparent $\mathrm{N}$ efficiency (milk $\mathrm{N}$ yield/N intake) responded positively to substitution of a protein source by canola meal suggesting an overall improvement in $\mathrm{N}$ metabolism of dairy cows.

Effect of diet composition and incubation time on feed indigestible neutral detergent fiber concentration in dairy cows. By Krizsan and Huhtanen, page 1715. A rapid and cost-effective method for analysis of indigestible neutral detergent fiber (NDF) by commercial laboratories has been developed from near infrared reflectance spectroscopy. Forage digestibility is precisely predicted from the NDF residues after 288-h ruminal in situ incubations. Reference samples would benefit from a shorter incubation time, and effects of diet composition on potential extent of NDF digestion from prolonged incubation periods have not been studied earlier. We conclude that indigestible NDF should be determined by 288 -h ruminal in situ incubations in cows consuming diets with a low proportion of concentrate to represent the feed fraction that is unavailable to the animal.

Effects of dietary forage sources on rumen microbial protein synthesis and milk performance in early lactating dairy cows. By Zhu et al., page 1727. Forage comprises more than half of a dairy cow's diet. Because of the differences in the physical, chemical, and digestion characteristics, forages have the potential to alter the supply of metabolizable nutrients to ruminants and affect lactation performance. When used as a forage source for lactating cows, corn stover had similar effects to Chinese wild rye grass on rumen microbial protein synthesis and milk production. A high proportion of alfalfa hay in the diet is beneficial for milk protein production through increasing microbial protein synthesis. This can be attributed to the available energy supply.

Effect of Lactobacillus inoculants and forage dry matter on the fermentation and aerobic stability of ensiled mixed-crop tall fescue and meadow fescue. By Guo et al., page 1735. This study evaluated the effects of Lactobacillus plantarum (LP) with or without Lactobacillus buchneri (LP $+\mathrm{LB})$ on the fermentation and aerobic stability of mixed tall fescue 
and meadow fescue silage ensiled at different dry matters. The LP treatment improved silage fermentation compared with untreated or LP+LB treated silages. Aerobic stability of grass mixes ensiled between 18 and $44 \%$ dry matter (DM) increased as DM increased. The LP and LP+LB inoculants improved aerobic stability of silages harvested between 18 and 44\% DM. These findings provide useful information about the effects of inoculants and DM on the fermentation and aerobic stability of ensiled cool-season grasses.

Biological components in a standardized derivative of bovine colostrum. By Sacerdote et al., page 1745. Products of different origin, time of collection, and activities fall in the general term of colostrum. In the present study, we describe the standardization of a bovine colostrum derivative and the characterization of its bioactive factors. We defined and set up an extraction and purification protocol from the most concentrated colostrum, which is that secreted in the first hours. The final lyophilized product was a sterile, pyrogen-free standardized derivative of bovine colostrum, maintaining most of the (several) bioactive factors at the basis of its many nutritional, modulatory, or therapeutic applications.

Effects of cobalt supplementation and vitamin $B_{12}$ injections on lactation performance and metabolism of Holstein dairy cows. By Akins et al., page 1755 . Dairy cows have reduced vitamin $\mathrm{B}_{12}$ status during early lactation, and cobalt supplementation may improve vitamin $\mathrm{B}_{12}$ status and lactation performance. The objective of this trial was to determine lactation performance of primiparous and multiparous dairy cows fed different levels and sources (inorganic and organic) of cobalt or injected weekly with vitamin $\mathrm{B}_{12}$. Milk and component yields were not improved by supplemental cobalt (either organic or inorganic) or vitamin $\mathrm{B}_{12}$ injections compared with a diet with no supplemental cobalt. Vitamin $\mathrm{B}_{12}$ contents of milk and liver were increased by cobalt supplementation and vitamin $\mathrm{B}_{12}$ injections.

Response profiles of enteric methane emissions and lactational performance during habituation to dietary coconut oil in dairy cows. By Hollmann et al., page 1769. Dietary coconut oil (CNO) is a potential feed ingredient to reduce enteric $\mathrm{CH}_{4}$ emissions from lactating dairy cows. Here, we evaluated enteric $\mathrm{CH}_{4}$ emissions, dry matter intake (DMI), and milk and milk fat yields of lactating dairy cows during habituation to dietary CNO. Feeding $2.7 \%$ or more CNO reduced $\mathrm{CH}_{4}$ emissions, reduced DMI, and depressed yields of milk fat and solids-corrected milk. With increasing CNO concentrations, these responses occurred sooner after treatment initiation. Onset of DMI depression and mitigation of enteric $\mathrm{CH}_{4}$ emitted per unit of DMI coin- cided with milk fat depression. Analysis of habituation to a dietary $\mathrm{CNO}$ challenge aids in the understanding of the effects of CNO.

Effects of coconut and fish oils on ruminal methanogenesis, fermentation, and abundance and diversity of microbial populations in vitro. By Patra and $Z u$, page 1782. This study investigated the comparative effects of coconut (CO) and fish oil (FO) on methanogenesis, fermentation, and microbial abundances and diversity using in vitro rumen mixed cultures containing 3.1 and $6.2 \mathrm{~mL} / \mathrm{L}$ of each oil. Both $\mathrm{CO}$ and FO decreased methane concentrations and feed digestibility to a similar extent, but the mode of inhibition and the effect on abundances and diversity of archaeal and bacterial populations differed between the oils. Total volatile fatty acid concentrations were unaffected, but $\mathrm{CO}$ reduced acetate percentage and acetate to propionate ratio and increased the percentages of propionate and butyrate to greater extents than did FO.

Glucagon-like peptide 2 therapy reduces negative effects of diarrhea on calf gut. By Connor et al., page 1793. Intestinal damage can cause diarrhea, reduce nutrient absorption, negatively affect animal growth, and adversely affect long-term animal productivity. As a model to evaluate the ability of glucagon-like peptide 2 (GLP-2) therapy to improve gut development and reduce intestinal damage caused by an infectious agent, coccidiosis was experimentally established in neonatal Holstein bull calves. Effects of intestinal nitrooxidative stress were reduced in GLP-2-treated infected calves versus untreated infected controls, indicating the potential usefulness of GLP-2 as a therapeutic for calf diarrhea.

Short communication: Effects of frequency of feed delivery and bunk space on the feeding behavior of limit-fed dairy heifers. By Greter et al., page 1803. The objective of this experiment was to determine the interaction between feed bunk space and frequency of feed provision on the feeding behavior patterns and growth of growing dairy heifers fed a limited amount. Sixteen Holstein dairy heifers were exposed to each of 4 treatments using a $4 \times 4$ Latin square design with a $2 \times 2$ factorial arrangement of treatments: providing a high-concentrate ration at $2.0 \%$ of body weight either once daily or twice daily in close succession $(2 \mathrm{~h}$ apart), and providing feed bunk space at either 0.40 or $0.29 \mathrm{~m} /$ heifer. Results indicated that providing limitfed dairy heifers their allotment of feed in one feeding per day increased time spent feeding and tended to improve weight gain. Further, providing $0.40 \mathrm{~m} /$ heifer feed bunk space increased weight gain, improved feed efficiency, and minimized individual variation in feeding time. 
Short communication: Intensive measurements of standing time of dairy calves housed in individual pens within a naturally ventilated, unheated nursery over different periods of the year. By Hill et al., page 1811. Intensive measurements of calf standing time in calves less than 2 mo of age found that Holstein calves averaged approximately 300 min/d standing with standing time increasing by approximately $0.5 \mathrm{~min} / \mathrm{d}$ with age and not differing with period of year. Standing time over the day was greatest during morning and afternoon feeding, intermediate during midday and evening, and least at night. Variation of standing time within period due to calf and variation across periods were not different.

Short communication: Milk output in llamas (Lama glama) in relation to energy intake and water turnover measured by an isotope dilution technique. By Riek et al., page 1815. Milk output was measured from water kinetics in llama dams using a stable hydrogen isotope at 4 different lactation stages: $3,10,18$, and 26 wk postpartum. We also recorded total water and energy intakes during that time. Our results show that the isotope dilution technique gives reliable results in terms of water turnover and thus milk output during lactation. Furthermore, energy intakes in llama dams during lactation are reported here for the first time and are expected to form the basis for future energy recommendations in lactating llamas.

Effects of Bos taurus autosome 9-located quantitative trait loci haplotypes on the disease phenotypes of dairy cows with experimentally induced Escherichia coli mastitis. By Khatun et al., page 1820. Quantitative trait loci (QTL) affecting mastitis incidence and mastitis-related traits, such as somatic cell count (SCC) exist in dairy cows. We induced Escherichia coli mastitis in Danish HolsteinFriesian cows carrying QTL haplotypes related to mastitis susceptibility on Bos taurus autosome 9. A low-susceptibility and a high-susceptibility QTL haplotype group of cows were examined for their clinical and paraclinical disease phenotype responses. Clinically, the high-susceptibility group tended to have a higher body temperature and heart rate during the acute disease stage than the low-susceptibility group did. Paraclinically, the low-susceptibility group had faster blood polymorphonuclear neutrophilic leukocyte recruitment and milk SCC recovery than the high-susceptibility group did. Hence, QTL haplotypes affected disease phenotype responses during E. coli mastitis.

Comparison of random regression and repeatability models to predict breeding values from test-day records of Norwegian goats. By Andonov et al., page 1834. For goats in Norway with about 5 records for daily milk yield and 3 for content traits per lactation, random regression models, producing a trajectory of breeding values across lactation, were shown to predict genetic merit more accurate than a repeatability model, allowing one breeding value for the entire lactation. Our recommendation was obtained by performing quality control through cross-validation; splitting the data and correlating excluded phenotypes in one dataset to the pedigree breeding value at the relevant day of lactation of the other dataset.

Evaluation of heat stress effects on production traits and somatic cell score of Holsteins in a temperate environment. By Hammami et al., page 1844. The anticipated change in climate is expected to have an effect on heat tolerance of dairy cows, and even cows in temperate regions are expected to be under heat stress. Thresholds for different thermal indices have to be identified and should be made available to breeders for herd management and genetic selection for heat tolerance.

Associations of acetyl-CoA carboxylase $\alpha$, stearoyl-CoA desaturase, and lipoprotein lipase genes with dairy traits in Alpine goats. By Crepaldi et al., page 1856. Milk yield and fat content have a great economic importance in goats. This species has a relevant role in marginal areas of Europe and in many developing countries. In the present work, associations between polymorphisms at 3 candidate genes and milk traits were investigated in Alpine goats farmed in Italy. The significant associations found could be exploited for the development of gene-assisted breeding programs in goat species.

Contribution of domestic production records, Interbull estimated breeding values, and single nucleotide polymorphism genetic markers to the single-step genomic evaluation of milk production. By Přibyl et al., page 1865. Molecular genetic information improved accuracy of selection of farm animals for production traits. A single-step procedure used direct nationwide phenotype data together with molecular genetic information both for genotyped and nongenotyped animals jointly in one evaluation. This methodology allows the combination of data from a domestic population and the global Interbull database, which improves prediction, especially in small populations.

Confirmation and discovery of maternal grandsires and great-grandsires in dairy cattle. By VanRaden et al., page 1874. Pedigree information that is unknown or incorrect can now be discovered or corrected using new genotyping tools. Breeders previously could identify parents and confirm those relationships using a few markers, or discover only the parents if they were genotyped with thousands of markers. Three 
methods to discover more distant ancestors were developed and compared. The most accurate method discovered $>90 \%$ of the correct maternal grandsires and great-grandsires from a list of $>10,000$ potential ancestor bulls. Confirmed and discovered maternal grandsires are now provided to breeders routinely.

Short communication: Analysis of health and survival in a population of Ontario Holstein heifer calves. By McCorquodale et al., page 1880. Calfhood disease and survival have severe economic consequences for dairy producers. The aim of the present study was to investigate health and survival and to identify factors that influence risk of mortality in a population of Ontario Holstein heifer calves. Among the various factors studied, season of birth, serum total protein, body weight of calves, bovine respiratory disease and the number of treatments a calf received influenced early calf mortality. Calf health and survival were found to be low heritable traits and estimates were associated with high standard errors. Further investigation and improved data collection are required to determine the full effect of genetics on health and survival in dairy heifer calves.

Short-term application of prestimulation and increased automatic cluster remover threshold affect milking characteristics of grazing dairy cows in late lactation. By Edwards et al., page 1886. The cluster-on time of individual cows is an important factor in determining herd milking duration. Results indicate that increasing automatic cluster remover thresholds can reduce individual cow cluster-on time by over $21 \%$ without compromising milk yield, milk composition, or indicators of udder health. In comparison, premilking stimulation increased the time cows were in the dairy and did not affect milk production.

Competitiveness of management-intensive grazing dairies in the mid-Atlantic region from 1995 to 2009. By Hanson et al., page 1894. For the small dairy operations examined in this study (none of which had more than 200 cows), management-intensive grazing (MIG) dairy farms were more profitable on per hundredweight, per cow, and per acre bases, and were no less profitable on a whole-farm basis. Confinement operators had higher gross income but expenses that exceeded those of MIG operators, so that MIG operators were at least as profitable. Profits of MIG operations were less variable as well, so that MIG operators faced less income risk.

Artificial insemination field data on the use of sexed and conventional semen in nulliparous Holstein heifers. By Healy et al., page 1905. Sexsorting reduces conception rates because the sorting process compromises fertilizing ability and is associated with a lower insemination dose. This study examines the effect of management and environmental variables on conception rates achieved using sexed and unsexed semen to breed dairy heifers. Breeding outcomes have been quantified in terms of sex ratio, gestation length, and twinning, abortion, and stillbirth rates. Sex preselection accelerates genetic gain, reduces dystocia, and improves herd biosecurity. Increased implementation of this technology would also address the problem of superfluous bull calves, a major welfare issue for the dairy industry.

Montbéliarde-sired crossbreds compared with pure Holsteins for dry matter intake, production, and body traits during the first 150 days of first lactation. By Hazel et al., page 1915. Dry matter intake and fat plus protein production of Montbéliarde-sired crossbred cows were not significantly different from pure Holstein cows during the first 150 days of first lactation. Montbéliarde $\times$ Holstein crossbred cows were similar to pure Holstein cows for hip height; however, Montbéliarde $\times$ (Jersey/Holstein) crossbred cows had significantly shorter hip height than both Montbéliarde $\times$ Holstein crossbred and pure Holstein cows. Because they carried more body condition, the Montbéliarde-sired crossbred cows had greater body weight than pure Holstein cows during early first lactation. For fertility, Montbéliarde-sired crossbred cows had significantly fewer days open than pure Holstein cows during first lactation. 